\title{
L-carnosine enhanced reproductive potential of the Saccharomyces cerevisiae yeast growing on medium containing glucose as a source of carbon
}

\author{
Magdalena Kwolek-Mirek • Mateusz Molon • \\ Pawel Kaszycki $\cdot$ Renata Zadrag-Tecza
}

Received: 2 December 2015/ Accepted: 30 March 2016/Published online: 4 April 2016

(C) The Author(s) 2016. This article is published with open access at Springerlink.com

\begin{abstract}
Carnosine is an endogenous dipeptide composed of $\beta$-alanine and L-histidine, which occurs in vertebrates, including humans. It has a number of favorable properties including buffering, chelating, antioxidant, anti-glycation and anti-aging activities. In our study we used the Saccharomyces cerevisiae yeast as a model organism to examine the impact of $\mathrm{L}$ carnosine on the cell lifespan. We demonstrated that Lcarnosine slowed down the growth and decreased the metabolic activity of cells as well as prolonged their generation time. On the other hand, it allowed for enhancement of the yeast reproductive potential and extended its reproductive lifespan. These changes may be a result of the reduced mitochondrial membrane potential and decreased ATP content in the yeast cells. However, due to reduction of the post-reproductive lifespan, L-carnosine did not have an influence on the total lifespan of yeast. In conclusion, L-carnosine does not extend the total lifespan of $S$. cerevisiae but rather
\end{abstract}

M. Kwolek-Mirek $(\bowtie) \cdot$ M. Molon · R. Zadrag-Tecza Department of Biochemistry and Cell Biology, Faculty of Biology and Agriculture, University of Rzeszow,

Zelwerowicza 4, 35-601 Rzeszow, Poland

e-mail: mkwolek@ur.edu.pl

P. Kaszycki

Unit of Biochemistry, Institute of Plant Biology and

Biotechnology, Faculty of Biotechnology and

Horticulture, University of Agriculture in Krakow, al. 29

Listopada 54, 31-425 Kraków, Poland it increases the yeast's reproductive capacity by increasing the number of daughter cells produced.

Keywords L-carnosine - Lifespan - Reproductive potential $\cdot$ Yeast $\cdot$ Mitochondrial membrane potential . ATP

\section{Introduction}

Carnosine ( $\beta$-alanyl-L-histidine) is a water-soluble dipeptide which occurs naturally in the millimolar range in mammals, including humans. The highest concentrations of carnosine are observed in the skeletal muscle tissue, central nervous system and cardiac muscle, with lower concentrations found in stomach, liver and kidney. It can also be found in muscles of fish, amphibians, reptiles and birds, but never in plants, fungi or other eukaryotes (Boldyrev et al. 2013). Carnosine has three ionisable groups: the amino group of $\beta$-alanine as well as the carboxylic group and the nitrogens of imidazole ring of $\mathrm{L}$ histidine. This chemical structure of carnosine determines its properties. The nitrogen atoms of carnosine imidazole ring $\left(\mathrm{p} K_{\mathrm{a}}=6.72\right)$ regulate the buffering activity of the dipeptide, which is particularly important in the skeletal and cardiac muscle. Carnosine also displays the metal ion chelating activity. It can form complexes with $\mathrm{Cu}^{2+}, \mathrm{Co}^{2+}, \mathrm{Ni}^{2+}, \mathrm{Cd}^{2+}$ and $\mathrm{Zn}^{2+}$, which have a wide range of biological relevance (Hill 
and Blikslager 2012; Mizuno et al. 2015; Mozdzan et al. 2005). Furthermore, carnosine is well-known for its antioxidant properties. It has both the ability to directly scavenge reactive oxygen species such as peroxynitrite and hypochlorite, and increase the content and/or regeneration of enzymatic and nonenzymatic antioxidants (Fontana et al. 2002; Hipkiss et al. 1998b; Kim et al. 2011; Klebanov et al. 1997). It was also confirmed that carnosine participates in prevention of the formation of advanced lipoxidation end-products (ALEs) and advanced glycation endproducts (AGEs). Carnosine is not only able to prevent protein carbonylation but can also react directly with protein carbonyl groups, producing protein-carbonylcarnosine adducts that prevent cross-linking to other unmodified proteins (Aldini et al. 2005; Brownson and Hipkiss 2000; Hipkiss et al. 1998a; Xie et al. 2013).

Under physiological conditions, the endogenous carnosine plays a crucial role in the skeletal and cardiac muscle as well as the neuronal tissue. In turn, the exogenous carnosine is considered a potential therapeutic agent for many diseases such as diabetes, ischemia/reperfusion damage, ocular diseases and neurological disorders (Alzheimer and Parkinson disease, schizophrenia and autistic spectrum disorders) (Aldini et al. 2005; Bellia et al. 2011; Boldyrev et al. 2013; Hipkiss 2007). Carnosine also reveals anti-cancer and anti-aging activities. It inhibits selectively the growth of transformed cell lines and tumour cells by suppressing cellular ATP generation (Iovine et al. 2012; Renner et al. 2010; Shen et al. 2014). In contrast, McFerland and Holliday (1994) demonstrated that carnosine could increase the lifespan as well as chronological age of cultured human diploid fibroblasts. In addition, it was found to rejuvenate already senescent cells giving them a more juvenile appearance (Holliday and McFarland 2000). Significant increase in the lifespan was also reported in peripheral blood derived human $\mathrm{CD} 4+\mathrm{T}$ cell clones after long-term culture with carnosine (Hyland et al. 2000). The anti-aging effect of carnosine has been described both for the cell lines and animal models. The study by Boldyrev group reported that carnosine supplemented to a standard diet attenuated the development of senile features and increased the lifespan in senescence-accelerated mice (Boldyrev et al. 1999; Gallant et al. 2000). It proved to increase significantly the number of spermatogonia and Sertoli cells in mice prone to accelerated aging (Gopko et al. 2005), and furthermore, extended the lifespan of male Drosophila melanogaster flies (Yuneva et al. 2002) and Brachionus manjavacas rotifers (Snell et al. 2012).

Studies of the influence of various chemical factors on the cellular or organismal level have been conducted on a wide range of model organisms. Among these, the yeast Saccharomyces cerevisiae is a commonly used organism to study the influence of such factors on the growth, lifespan and aging process (Krzepilko et al. 2004; Lam et al. 2010; Wu et al. 2014). This yeast has also been used to study the effect of carnosine. Cartwright et al. (2012) showed that carnosine exhibited either inhibitory, or stimulatory effects on yeast cells, depending on the carbon source in the growth medium.

The aim of this study was to investigate the effect of L-carnosine on the rate of growth, reproductive potential, lifespan and metabolic activity of $S$. cerevisiae cells cultivated in the medium supplemented with glucose as a source of carbon. We also tested the cellular ATP content and mitochondrial membrane potential as affected by the presence of the studied dipeptide.

\section{Materials and methods}

Yeast strain, media and growth conditions

In the study a wild-type strain BY4742 MAT $\alpha$ his3 41 leu $2 \Delta 0$ lys $2 \triangle 0$ ura $\triangle 0$ (EUROSCARF) was used. The yeast was grown in the standard liquid YPD medium (1\% Yeast Extract, $1 \%$ Yeast Bacto-Peptone, $2 \%$ glucose) on a rotary shaker at $150 \mathrm{rpm}$, or on the solid YPD medium containing $2 \%$ agar, at the temperature of $28^{\circ} \mathrm{C}$.

\section{Determination of cell growth}

Liquid yeast cultures $\left(5 \times 10^{6}\right.$ cells $/ \mathrm{ml}$ in a total volume of $200 \mu \mathrm{l}$ of cells) with or without $20 \mathrm{mM} \mathrm{L-}$ carnosine were cultivated in a Heidolph incubator 1000 at $1200 \mathrm{rpm}$ at $28{ }^{\circ} \mathrm{C}$. Their growth was monitored turbidimetrically at $600 \mathrm{~nm}$ in an Anthos 2010 type 17,550 microplate reader for $12 \mathrm{~h}$ (measured every $1 \mathrm{~h}$ ), then after 24 and $48 \mathrm{~h}$. The relative growth rate was calculated at the exponential growth phase using an appropriate formula (Hall et al. 2014). All the data represent mean values obtained in four independent experiments. 
Determination of cell lifespan

Saccharomyces cerevisiae cell lifespan was determined as described previously (Minois et al. 2005; Zadrag et al. 2008). Overnight yeast cultures were dropped onto the YPD plates with the solid medium containing Phloxine B at the concentration of $10 \mu \mathrm{l} /$ $\mathrm{ml}$. During the manipulation, the plates were kept at $28{ }^{\circ} \mathrm{C}$ for $16 \mathrm{~h}$ and at $4{ }^{\circ} \mathrm{C}$ overnight. The reproductive potential (the number of buds produced), reproductive lifespan (the time during which a yeast cell is able to reproduce), post-reproductive lifespan (yeast cell life duration after the cessation of reproduction) and total lifespan (sum of the reproductive and postreproductive lifespans) were analysed for forty single cells in each experiment. The data represent mean values from two separate experiments.

Incubation and growth conditions

Yeast cells from the exponential phase culture were centrifuged, washed with sterile water and suspended either to the final density of $10^{8}$ cells $/ \mathrm{ml}$ in $100 \mathrm{mM}$ phosphate buffer with pH 7.0 containing $0.1 \%$ glucose and $1 \mathrm{mM}$ EDTA, or to the final density of $5 \times 10^{6}$ cells $/ \mathrm{ml}$ in the YPD medium; in both cases with or without addition of $20 \mathrm{mM} \mathrm{L}$-carnosine. After 1, 3 and $6 \mathrm{~h}$ of incubation in buffer or 3, 6, 12, 24, 48 and $72 \mathrm{~h}$ of growth in YPD medium, the cells were pelleted by centrifugation, then washed twice with sterile water and used for further analysis.

\section{Assessment of mitochondrial membrane potential}

The mitochondrial membrane potential was assessed with both rhodamine 123 and rhodamine B hexyl ester according to the manufacturer's protocol (Molecular Probes). The cells after growth in the presence or absence of L-carnosine were suspended either in $50 \mathrm{mM}$ citrate buffer with $\mathrm{pH} 5.0$ containing $2 \%$ glucose, or in $10 \mathrm{mM}$ HEPES buffer with $\mathrm{pH} 7.4$ containing $5 \%$ glucose, for the case of rhodamine 123 and rhodamine B hexyl ester staining, respectively. Rhodamine 123 was added to the final concentration of $5 \mu \mathrm{M}$, and after $15 \mathrm{~min}$ of incubation the fluorescence of the cell suspension was measured using the TECAN Infinite 200 microplate reader at $\lambda_{\mathrm{ex}}=505$ $\mathrm{nm}$ and $\lambda_{\mathrm{em}}=534 \mathrm{~nm}$. The data represent mean values obtained upon three independent experiments.
The mitochondrial network was visualised by fluorescence microscopy Olympus BX-51 using $100 \mathrm{nM}$ rhodamine $\mathrm{B}$, the fluorescent dye in which the emission is dependent on the mitochondrial membrane potential, at $\lambda_{\mathrm{ex}}=555 \mathrm{~nm}$ and $\lambda_{\mathrm{em}}=579 \mathrm{~nm}$. The photographs present a typical result of a duplicate experiment.

Assessment of the cellular ATP content

The level of ATP in the yeast cells was assessed with BacTiter-Glo $^{\mathrm{TM}}$ Microbial Cell Viability Assay according to the manufacturer's protocol (Promega). The cells after incubation or growth in the presence or absence of L-carnosine were suspended in a $100 \mathrm{mM}$ phosphate buffer with $\mathrm{pH} 7.0$ containing $0.1 \%$ glucose and $1 \mathrm{mM}$ EDTA. A sample of cell suspension with a density of $10^{6}$ cells $/ \mathrm{ml}$ was used for determination purposes. The luminescent signal, as proportional to the amount of ATP present, was recorded after 5 min using the TECAN Infinite 200 microplate reader. The cellular ATP content was calculated from the standard curve. The data represent mean values from four independent experiments.

Assessment of the metabolic activity

The metabolic activity of the yeast cells was assessed with FUN-1 according to the manufacturer's protocol (Molecular Probes). The cells after incubation or growth in the presence or absence of L-carnosine were suspended in a $10 \mathrm{mM}$ HEPES buffer with $\mathrm{pH} 7.2$ containing $2 \%$ glucose. The metabolic activity of cells was estimated with $0.5 \mu \mathrm{M}$ FUN-1. The fluorescence of the cell suspension was measured after 15 min using the TECAN Infinite 200 microplate reader at $\lambda_{\mathrm{ex}}=480 \mathrm{~nm}$ and $\lambda_{\mathrm{em}}=500-650 \mathrm{~nm}$. The metabolic activity of cells was expressed as a change in ratio of red $(\lambda=575 \mathrm{~nm})$ to green $(\lambda=535 \mathrm{~nm})$ fluorescence. The data represent mean values from four independent experiments.

Statistical analysis

Data are presented as mean values \pm standard deviation (SD). The statistical analysis was performed using the SPSS 21.0 software. The statistical significance of the differences between the means of treated sample compared to untreated control was estimated 
using the $t$ test for independent samples. The differences between samples obtained after various time of incubation/growth with or without addition of Lcarnosine were evaluated using one-way ANOVA and the Dunnet post hoc test. Homogeneity of variance was checked using Levene's test. Values were considered significant if $p<0.05$.

\section{Results}

L-carnosine slows down the growth rate of yeast cells

Cartwright et al. (2012) have shown that L-carnosine decreased the growth of yeast cells in a medium containing a fermentable carbon source in a dosedependent manner. The results in Fig. 1 confirm that the addition of $20 \mathrm{mM} \mathrm{L}$-carnosine to the liquid
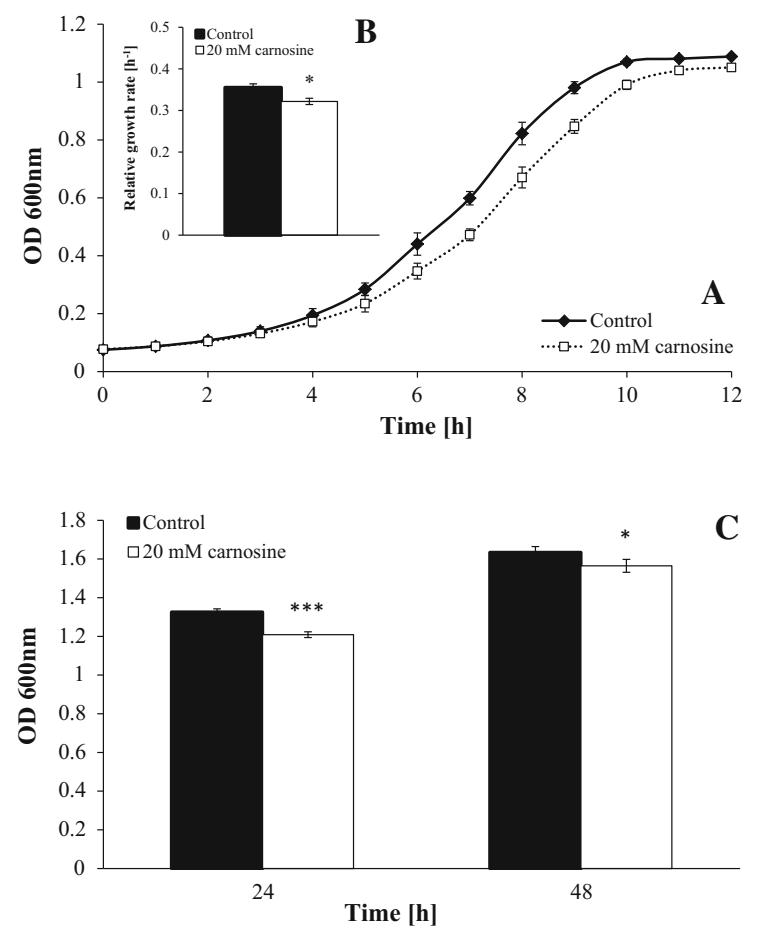

Fig. 1 Effect of L-carnosine on growth of the BY4742 strain in liquid YPD media. Kinetics of growth was monitored turbidimetrically at $600 \mathrm{~nm}$ every $1 \mathrm{~h}$ for $12 \mathrm{~h}$ (a), and after 24 and $48 \mathrm{~h}$ (c). The relative growth rate (b) was calculated using appropriate formula. Data are presented as mean \pm SD from four independent experiments. $* p<0.05$; $* * * p<0.001$ as compared to the untreated control medium containing $2 \%$ glucose slowed down the growth of the BY4742 strain yeast cells. This effect was visible both for the exponential phase of growth (Fig. 1a) and after 24 and $48 \mathrm{~h}$ of culture (Fig. 1c). We also observed a statistically significant decrease of the relative growth rate (Fig. 1b) and an increase in the average generation time as determined based on the growth curve by approx. $9 \%$ (data not shown) in the case of yeast cells exposed to L-carnosine, compared to the untreated control.

L-carnosine extends the reproductive potential but not the total lifespan of yeast cells

The reproductive potential and the total lifespan of yeast cells are not synonymous. The reproductive potential is defined as the number of daughter cells produced by a single mother cell during its life while the total lifespan is means the cell life duration including both the reproductive and post-reproductive phases. Therefore, we examined the effect of $20 \mathrm{mM}$ L-carnosine on these two parameters of the BY 4742 yeast. L-carnosine significantly enhanced the reproductive potential of yeast cells, increasing the average number of daughters produced from 20 to 26 (Fig. 2a; Table 1). The positive effect of $\mathrm{L}$-carnosine was even more pronounced when the time-parameter was taken into account. An increase in the average generation time (determined based on the reproductive lifespan) was observed and thus the average reproductive lifespan was extended by approx. 16 and $49 \%$, respectively (Fig. 2b; Table 1). On the other hand, Lcarnosine was found to reduce the average postreproductive lifespan of yeast cells (during this time the cells were still alive but not able to reproduce) by approx. $46 \%$ (Fig. 2c; Table 1). Interestingly, the total lifespan in the case of both the control conditions and the cells exposed to L-carnosine was almost the same (Fig. 2d; Table 1). These results show that Lcarnosine is not able to extend the total lifespan of yeast cells; however, it can significantly influence their reproductive potential.

L-carnosine decreases the cellular energy level and the metabolic activity

The yeast $S$. cerevisiae receives energy from the fermentation process in the presence of fermentable carbon sources such as glucose or from the 

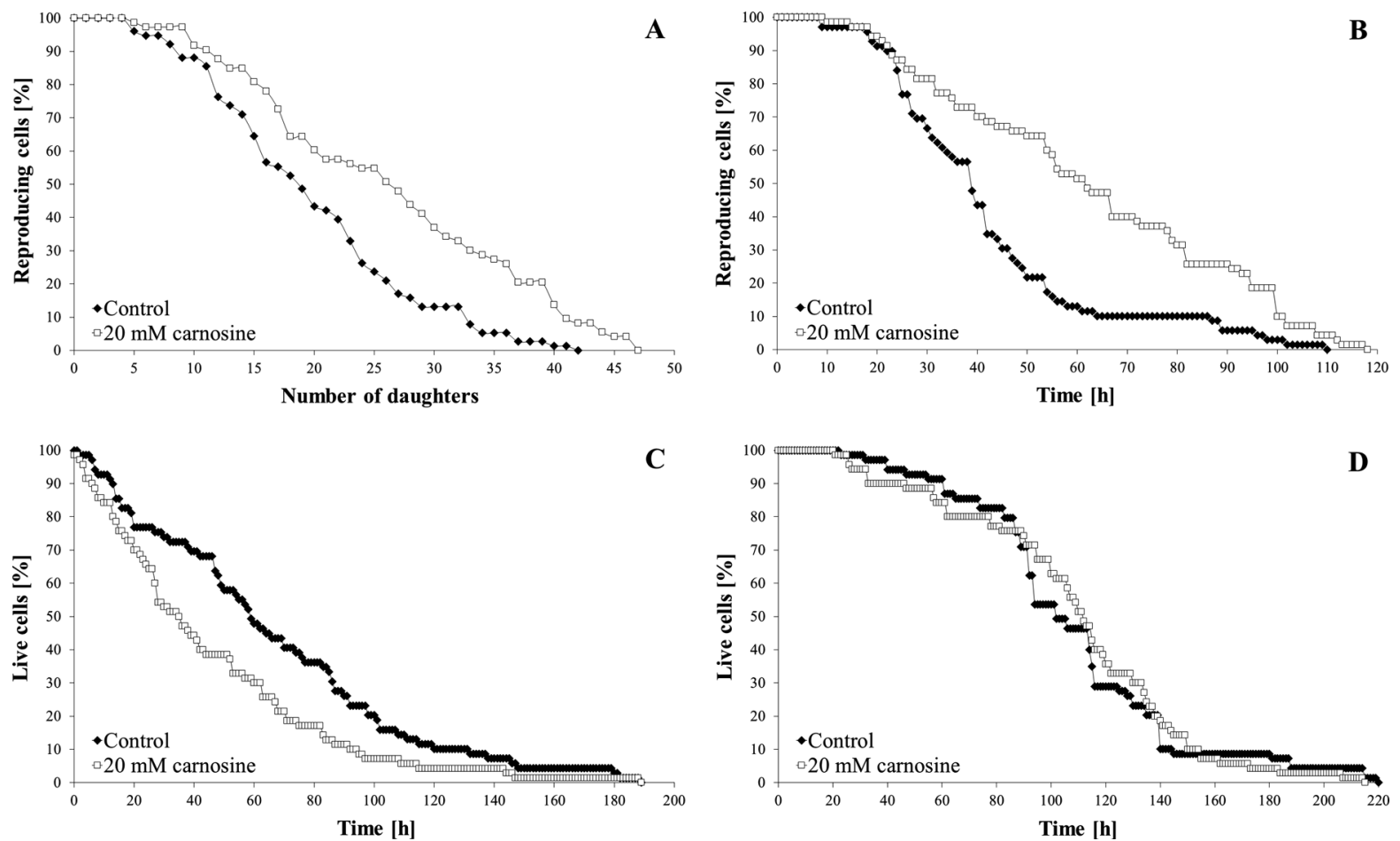

Fig. 2 Reproductive potential (number of generations; a), reproductive lifespan (b), post-reproductive lifespan (c) and total lifespan (d) of the BY4742 strain after cultivation on solid

YPD media with or without addition of $20 \mathrm{mM}$ L-carnosine. Data represent mean values from two independent experiments

Table 1 Reproductive potential (number of daughter cells produced), reproductive lifespan, post-reproductive lifespan and total lifespan of BY4742 strain after cultivation on YPD

media supplemented with $20 \mathrm{mM}$ L-carnosine, control-no carnosine supplementation

\begin{tabular}{lclcr}
\hline & $\begin{array}{l}\text { Reproductive potential } \\
\text { Number of daughter }\end{array}$ & $\begin{array}{l}\text { Reproductive lifespan } \\
\text { Time }(\mathrm{h})\end{array}$ & $\begin{array}{l}\text { Post-reproductive lifespan } \\
\text { Time (h) }\end{array}$ & $\begin{array}{l}\text { Total lifespan } \\
\text { Time }(\mathrm{h})\end{array}$ \\
\hline Control & $20 \pm 8.4$ & $42.1 \pm 21.8$ & $66 \pm 45$ & $108.1 \pm 40.6$ \\
$20 \mathrm{mM}$ L-carnosine & $25.7 \pm 11.7^{* *}$ & $62.6 \pm 29.7^{* * *}$ & $45.2 \pm 38.1^{* *}$ & $107.8 \pm 42.2$
\end{tabular}

Data are presented as mean values $\pm \mathrm{SD}$ obtained from all the cells tested during two independent experiments (80 cells)

** $p<0.01$; *** $p<0.001$ as compared to the untreated control using a $t$ test

aerobic respiration in the presence of non-fermentable carbon sources such as glycerol. Therefore, mitochondria are an important cellular energy centre, and their morphology and number may change under different conditions and along with age. The effect of $20 \mathrm{mM}$ L-carnosine on the mitochondrial membrane potential of the BY4742 cells was examined after short- and long-term culture. We observed that mitochondrial membrane potential after 3, 6 and $12 \mathrm{~h}$ of cultivation with $\mathrm{L}$-carnosine remained at a relatively constant level, significantly lower than in the case of the untreated control (Fig. 3a). The level of mitochondrial membrane potential was almost the same regardless the time of cultivation (sample after 24 and 48 h) (Fig. 3a, b). Moreover, L-carnosine did not change the morphology of mitochondria and only slightly affected the development of mitochondrial network (Fig. 3b).

The ATP yeast cell content is directly related to the level of glucose in the medium and the mitochondrial 


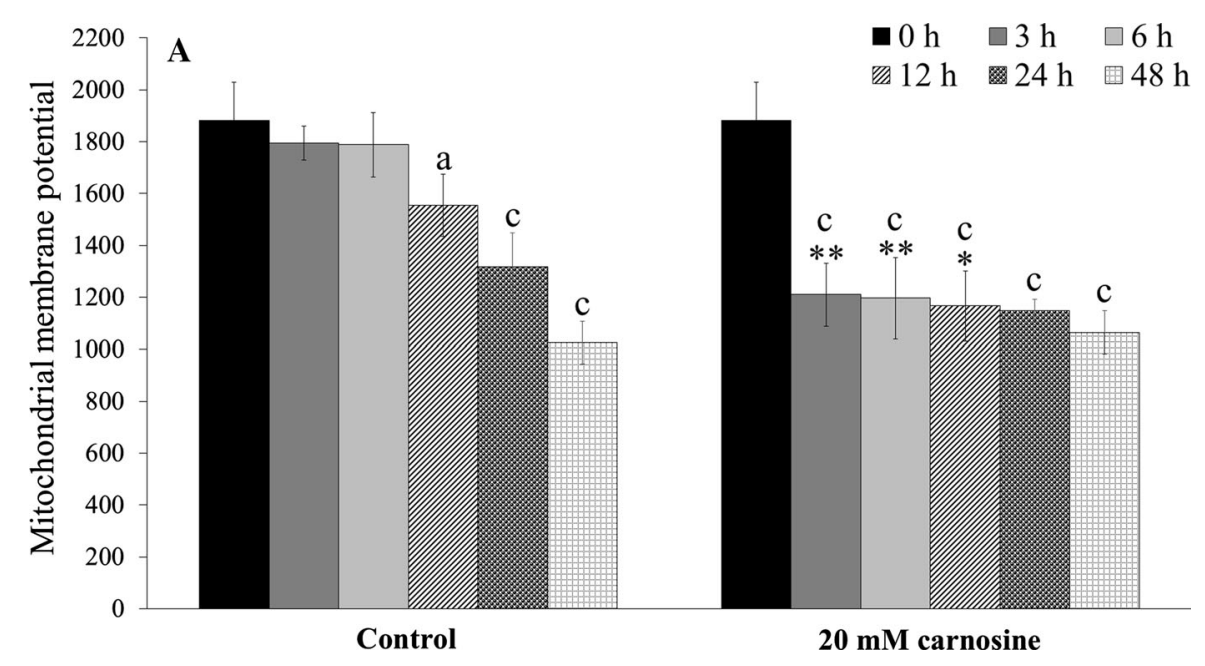

Fig. 3 Mitochondrial membrane potential of the BY4742 yeast cells after cultivation in YPD media with or without addition of $20 \mathrm{mM}$ L-carnosine. The cells were stained both with rhodamine 123 (a) and rhodamine B (b). Data are presented as mean \pm SD from three independent experiments. ${ }^{*} p<0.05$; $* * p<0.01$;

activity. $20 \mathrm{mM}$ L-carnosine decreased the level of ATP, but only after a short time of incubation/culture (Fig. 4a, b). In turn, after 24, 48 and $72 \mathrm{~h}$ of cultivation an opposite reaction was observed in the presence of $\mathrm{L}-$ carnosine: the cellular ATP content was significantly higher in comparison to the untreated control (Fig. 4b).

The cellular energy level has an impact on the vitality and viability of cells. Therefore, the effect of $20 \mathrm{mM}$ L-carnosine on the metabolic activity of the BY4742 cells was determined using a FUN-1 stain. Lcarnosine decreased the metabolic activity, both after a short time of incubation ( 3 and $6 \mathrm{~h}$ ) and after a longterm culture (12, 24, 48 and 72 h). For each case studied these values were significantly lower compared to the untreated control (Fig. 4c, d). These results show that L-carnosine can alter the cellular energy level, thereby reducing the metabolic activity of cells.

\section{Discussion}

The search for compounds that improve quality and prolong the time of human life has been conducted for many years in numerous laboratories around the world. Carnosine appears as one of such type of compounds with potential anti-aging properties.
*** $p<0.001$ as compared to the untreated control. Letters $a, b$ and $c$ on the graph indicate differences between the initial and subsequent times of experiment at $p<0.05 ; p<0.01$; $p<0.001$, respectively

Previous studies show that L-carnosine could increase the lifespan as well as chronological age of human fibroblasts (Holliday and McFarland 2000; McFarland and Holliday 1994) and extend the lifespan of selected animals such as mice (Boldyrev et al. 1999; Gallant et al. 2000), D. melanogaster flies (Yuneva et al. 2002) and B. manjavacas rotifers (Snell et al. 2012). The $S$. cerevisiae yeast is commonly used as a model organism to study the influence of various factors on the growth, lifespan and aging process (Krzepilko et al. 2004; Lam et al. 2010; Wu et al. 2014). For the studies concerning carnosine, usefulness of the $S$. cerevisiae is especially important because as a fungus this yeast does not produce L-carnosine and its metabolites, which enables us to examine the effect of extracellular L-carnosine on the cells using various doses and growth conditions. S. cerevisiae was first subjected to the research on properties of carnosine by Cartwright et al. (2012). Their study showed that Lcarnosine decreased the yeast growth in media containing fermentable carbon sources in a dose-dependent manner. This effect was more pronounced in the case of glucose than mannose, galactose or fructose. Lcarnosine did not have an inhibitory effect on the growth on the contrary provoked significant increase in the growth rate of yeast in media with nonfermentable carbon sources in the presence of oxygen. The results showed that L-carnosine decreased 

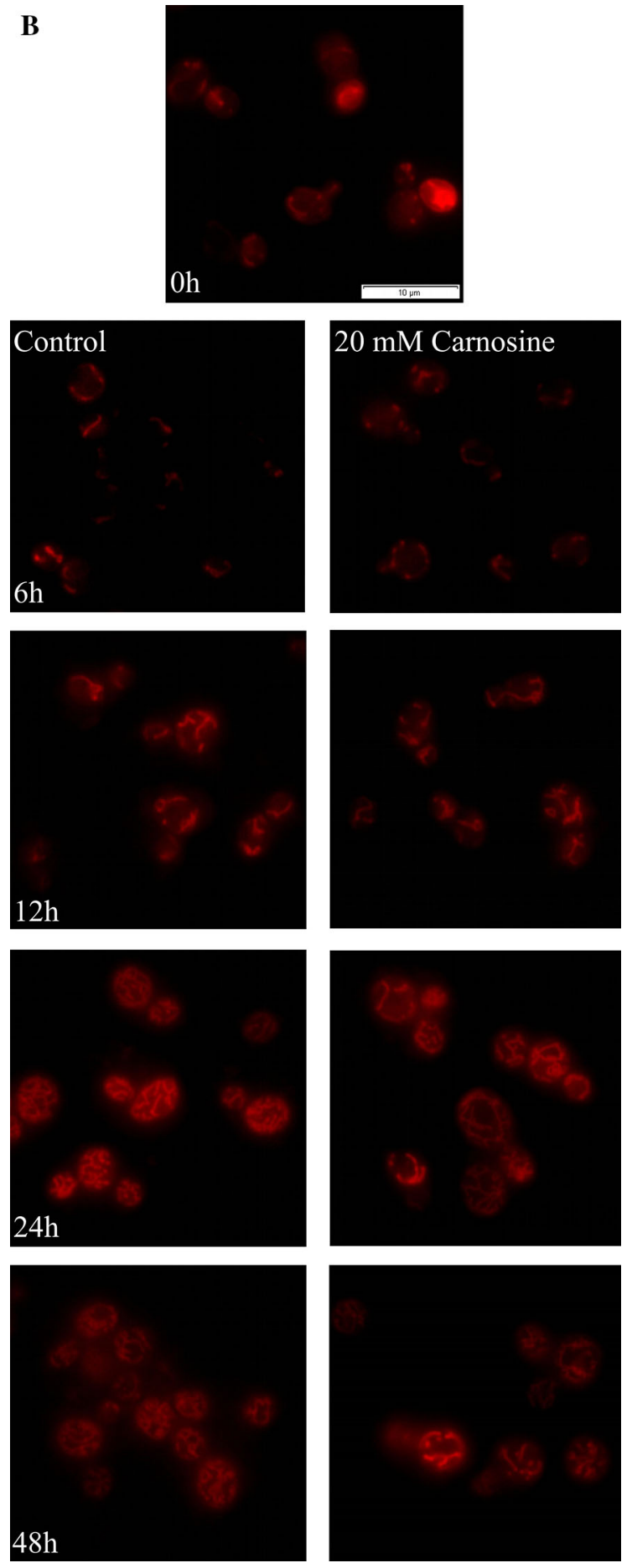

Fig. 3 continued

viability of cells but only under reduced oxidative phosphorylation conditions. Our results confirm that Lcarnosine slows down the growth of yeast on the YPD medium, decreases the relative growth rate and increases the generation time (Fig. 1a-c). However, the observed growth rate changes are the result of slowdown of cell reproduction cycle rather than the cell death.

Natural compounds with anti-aging properties are discovered relatively rare. Literature data indicate that L-carnosine may indeed exhibit such features (Boldyrev et al. 1999; McFarland and Holliday 1994; Snell et al. 2012; Yuneva et al. 2002). As the available references do not seem to fully confirm the case of the yeast S. cerevisiae (Cartwright et al. 2012; Fig. 1), we decided to investigate the effect of L-carnosine on individual cells of the mentioned species during the culture on solid YPD medium. Our study proves that $20 \mathrm{mM}$ L-carnosine significantly enhanced the reproductive cell potential (the number of buds produced, which in the literature is termed as a replicative lifespan) and extended the reproductive lifespan (the time during which a yeast cell is able to reproduce) of the BY4742 strain (Fig. 2a, b; Table 1). In the literature, only few compounds were reported as having the ability to increase the reproductive potential of yeast, such as resveratrol (Howitz et al. 2003), ascorbate (Krzepilko et al. 2004), diazaborine (Steffen et al. 2008) and ibuprofen (He et al. 2014). More information can be found regarding the effects of various factors on prolonging the chronological lifespan of yeast (Georgieva et al. 2015; Nakaya et al. 2014; Rockenfeller et al. 2015; Wanke et al. 2008; Wu et al. 2014). However, one should not compare these two types of experiments since the chronological lifespan and the total lifespan are not the same. Yeast cells do not end their life immediately after the reproduction phase; therefore, an analysis of the postreproductive phase makes it possible to determine their total lifespan. Our results indicate that $20 \mathrm{mM} \mathrm{L}^{-}$ carnosine reduced the average post-reproductive lifespan of yeast (Fig. 2c; Table 1). It follows that the addition of L-carnosine extends the reproductive lifespan and thereby shortens the post-reproductive lifespan of yeast. The negative correlation between the post-reproductive and reproductive lifespans was presented in our previous study for a number of yeast strains (Molon et al. 2015; Zadrag-Tecza et al. 2013). Here, the total lifespan was shown to be almost the same, both for the case of the control and the cells exposed to L-carnosine (Fig. 2d; Table 1). These results prove that L-carnosine has no pro-longevity 

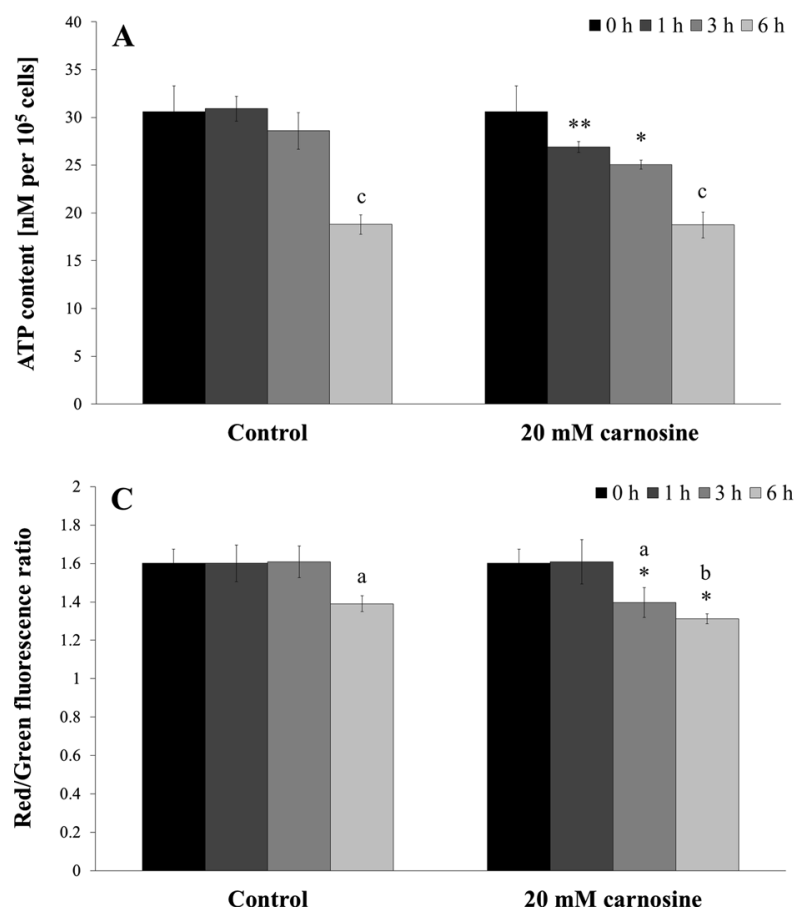

Fig. 4 Effect of L-carnosine on ATP content and metabolic activity of the BY4742 yeast cells. Cellular ATP content (a, b) determined using BactTiter-Glo ${ }^{\mathrm{TM}}$ Microbial Cell Viability Assay and metabolic activity (c, d) using FUN-1 stain. a, $\mathbf{c}$ results for short time of incubation, $\mathbf{b}, \mathbf{d}$ results for long-term of

effect because it does not extend the total yeast lifespan but rather causes an increase in its reproductive potential.

Enhancing the reproductive potential of the yeast treated with L-carnosine may be associated with the regulation of energy metabolism. Previous studies report that L-carnosine inhibits the ATP production and thus reduces the proliferative capacity of cancer cells (Iovine et al. 2012; Renner et al. 2010; Shen et al. 2014). Furthermore, Cartwright et al. (2012) demonstrated that L-carnosine caused changes in the metabolic activity of the yeast grown on the fermentable carbon source. Addition of L-carnosine significantly affected the heat output profiles of the cultures, measured using on-line flow microcalorimetry, in a dose-dependent manner (Cartwright et al. 2012). Based on our work, it is clearly seen that $20 \mathrm{mM}$ L-carnosine decreases mitochondrial membrane potential during the culture on YPD medium compared to the control, but only in the exponential phase of growth. The differences in the mitochondrial
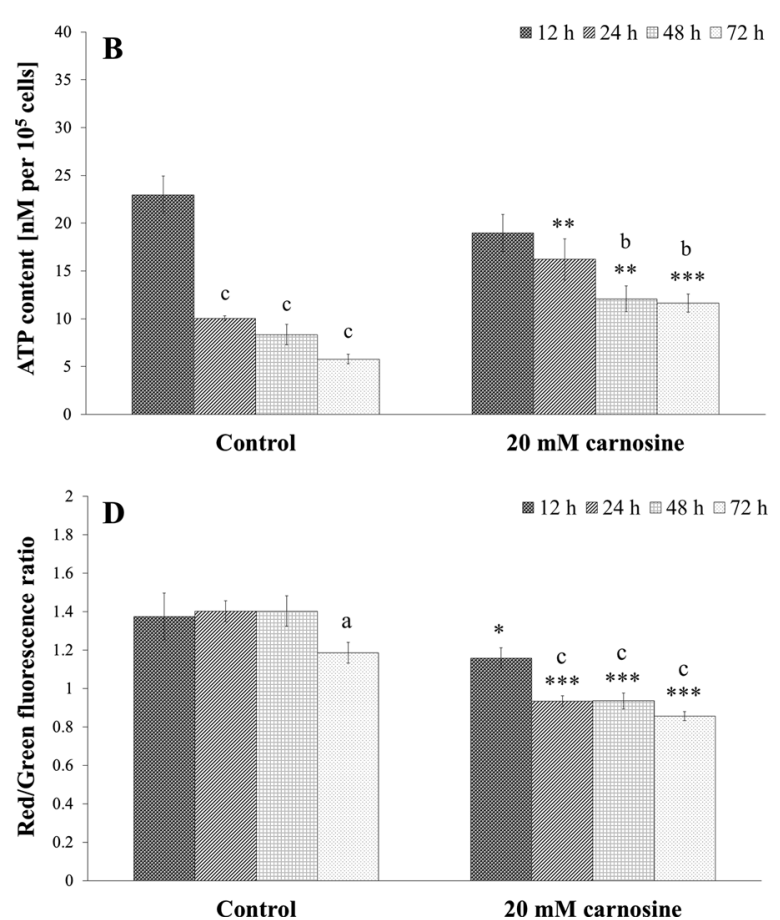

culture. Data are presented as mean \pm SD from four independent experiments. * $p<0.05$; ** $p<0.01$; *** $p<0.001$ as compared to untreated control. Letters $a, b$ and $c$ on the graph indicate differences between the initial and subsequent times of experiment at $p<0.05 ; p<0.01 ; p<0.001$, respectively

membrane potential are not visible in the stationary phase of growth when most of the glucose supply has been consumed and the yeast cells have transferred to the aerobic respiration (Fig. 3a). A mild decrease in the mitochondrial membrane potential is considered beneficial for cells and whole organism (Knorre and Severin 2012). This has been confirmed by the results of Barros et al. (Barros et al. 2004) with the use of low doses of protonophore dinitrophenol which caused an increase in the number of daughters produced (replicative lifespan). The proposed mechanism assumes that lowering the mitochondrial membrane potential may lead to preventing mitochondrial production of reactive oxygen species (ROS) but also may activate the retrograde response, which by transcriptional changes can result in an increase of the lifespan (Miceli et al. 2011).

We also observed a significant reduction of the ATP content in the cells after short incubation time in the presence of $20 \mathrm{mM}$ L-carnosine (Fig. 4a, b). In turn, after 24,48 and $72 \mathrm{~h}$ of culture we observed an 
opposite reaction and a significantly higher level of cellular ATP compared to the control (Fig. 4b). This effect may be a result of lower cell energy requirements in the presence of L-carnosine, its protective properties or amino acid supplementation deriving from carnosine metabolism. Such an increase in the ATP level enables the cells to increase the number of daughters produced (reproductive potential), and in terms of the time of a single reproduction cycle, to extend the reproductive lifespan (Fig. 2a, b; Table 1). The observed changes in ATP levels during prolonged culture can result from a changes in a way of energy production by yeast cells. At a high level of glucose it dominates the fermentative metabolism, which is changed into a respiration after consumption the majority of available glucose. The initial decrease in ATP level may result from the fact, that the addition of L-carnosine reduces the ATP production from glycolysis, as is demonstrated in the case of tumour cells (Renner et al. 2010). In turn the observed increase in the ATP level occurs after diauxic shift, where metabolism becomes aerobic. However, a higher level of ATP (which was still very low) made it impossible to maintain metabolic activity of the cells at a constant level (Fig. 4d). The addition of $20 \mathrm{mM}$ L-carnosine decreased the metabolic activity both at the exponential and stationary growth phases as compared to the control (Fig. 4c, d). These results confirm the earlier observations of Cartwright et al. (2012).

In summary, L-carnosine can change the cellular ATP content by decreasing the intensity of glycolysis which results in a reduction of the mitochondrial membrane potential, a decrease in the metabolic activity of cells and the extended time of generation. Reduction of the energy level in the cells leads to the enhanced reproductive potential and extended reproductive lifespan of yeast. As a regulator of cell energy metabolism, L-carnosine causes an efficient increase in the reproductive capacity as typically observed in the case of caloric restriction and repression of the enhanced gluconeogenesis (Evans et al. 2010; Hachinohe et al. 2013; Lin et al. 2002; Medvedik et al. 2007; Wierman and Smith 2014). On the other hand, we must not forget that due to reduction of the postreproductive lifespan the effect of L-carnosine on the total lifespan of yeast is not significant. L-carnosine does not extend the lifetime of a single yeast cell but can rather increase the chances for population survival by increasing the number of offspring.
Funding The study was partially supported by the Polish National Science Centre Grant No. 2013/09/B/NZ3/01352 and by the research fellowship within the project "University of Rzeszow-modernity and future of region" funded from the European Social Fund, Human Capital, National Cohesion Strategy (Contract No. UDA-POKL.04.01.01-00-068/10-00).

\section{Compliance with ethical standards}

Conflict of interest The authors declare that they have no conflict of interest.

Open Access This article is distributed under the terms of the Creative Commons Attribution 4.0 International License (http:// creativecommons.org/licenses/by/4.0/), which permits unrestricted use, distribution, and reproduction in any medium, provided you give appropriate credit to the original author(s) and the source, provide a link to the Creative Commons license, and indicate if changes were made.

\section{References}

Aldini G, Facino RM, Beretta G, Carini M (2005) Carnosine and related dipeptides as quenchers of reactive carbonyl species: from structural studies to therapeutic perspectives. BioFactors 24:77-87

Barros MH, Bandy B, Tahara EB, Kowaltowski AJ (2004) Higher respiratory activity decreases mitochondrial reactive oxygen release and increases life span in Saccharomyces cerevisiae. J Biol Chem 279:49883-49888. doi:10.1074/jbc.M408918200

Bellia F, Vecchio G, Cuzzocrea S, Calabrese V, Rizzarelli E (2011) Neuroprotective features of carnosine in oxidative driven diseases. Mol Asp Med 32:258-266. doi:10.1016/j. mam.2011.10.009

Boldyrev AA, Gallant SC, Sukhich GT (1999) Carnosine, the protective, anti-aging peptide. Biosci Rep 19:581-587

Boldyrev AA, Aldini G, Derave W (2013) Physiology and pathophysiology of carnosine. Physiol Rev 93:1803-1845. doi:10.1152/physrev.00039.2012

Brownson C, Hipkiss AR (2000) Carnosine reacts with a glycated protein. Free Radic Biol Med 28:1564-1570

Cartwright SP, Bill RM, Hipkiss AR (2012) L-carnosine affects the growth of Saccharomyces cerevisiae in a metabolismdependent manner. PLoS One 7:e45006. doi:10.1371/ journal.pone.0045006

Evans C, Bogan KL, Song P, Burant CF, Kennedy RT, Brenner $\mathrm{C}$ (2010) NAD + metabolite levels as a function of vitamins and calorie restriction: evidence for different mechanisms of longevity. BMC Chem Biol 10:2. doi:10.1186/14726769-10-2

Fontana M, Pinnen F, Lucente G, Pecci L (2002) Prevention of peroxynitrite-dependent damage by carnosine and related sulphonamido pseudodipeptides. Cell Mol Life Sci 59:546-551

Gallant S, Semyonova M, Yuneva M (2000) Carnosine as a potential anti-senescence drug. Biochemistry (Mosc) 65:866-868 
Georgieva M, Moyankova D, Djilianov D, Uzunova K, Miloshev G (2015) Methanol extracts from the resurrection plant Haberlea rhodopensis ameliorate cellular vitality in chronologically ageing Saccharomyces cerevisiae cells. Biogerontology 16:461-472. doi:10.1007/s10522-015-9566-Z

Gopko AV, Kulibin AY, Semenova ML, Zakhidov ST (2005) Carnosine-induced changes in the development of spermatogenic epithelial cells in senescence accelerated SAM mice. Bull Exp Biol Med 140:235-237

Hachinohe M, Yamane M, Akazawa D, Ohsawa K, Ohno M, Terashita Y, Masumoto H (2013) A reduction in age-enhanced gluconeogenesis extends lifespan. PLoS One 8:e54011. doi:10.1371/journal.pone.0054011

Hall BG, Acar H, Nandipati A, Barlow M (2014) Growth rates made easy. Mol Biol Evol 31:232-238. doi:10.1093/ molbev/mst 187

He C et al (2014) Enhanced longevity by ibuprofen, conserved in multiple species, occurs in yeast through inhibition of tryptophan import. PLoS Genet 10:e1004860. doi:10.1371/ journal.pgen. 1004860

Hill TL, Blikslager AT (2012) Effect of a zinc L-carnosine compound on acid-induced injury in canine gastric mucosa ex vivo. Am J Vet Res 73:659-663. doi:10.2460/ajvr.73.5. 659

Hipkiss AR (2007) Could carnosine or related structures suppress Alzheimer's disease? J Alzheimers Dis 11:229-240

Hipkiss AR et al (1998a) Pluripotent protective effects of carnosine, a naturally occurring dipeptide. Ann N Y Acad Sci 854:37-53

Hipkiss AR, Worthington VC, Himsworth DT, Herwig W (1998b) Protective effects of carnosine against protein modification mediated by malondialdehyde and hypochlorite. Biochim Biophys Acta 1380:46-54

Holliday R, McFarland GA (2000) A role for carnosine in cellular maintenance. Biochemistry (Mosc) 65:843-848

Howitz KT et al (2003) Small molecule activators of sirtuins extend Saccharomyces cerevisiae lifespan. Nature 425:191-196. doi:10.1038/nature01960

Hyland P, Duggan O, Hipkiss A, Barnett C, Barnett Y (2000) The effects of carnosine on oxidative DNA damage levels and in vitro lifespan in human peripheral blood derived CD4+ T cell clones. Mech Ageing Dev 121: 203-215

Iovine B, Iannella ML, Nocella F, Pricolo MR, Bevilacqua MA (2012) Carnosine inhibits KRAS-mediated HCT116 proliferation by affecting ATP and ROS production. Cancer Lett 315:122-128. doi:10.1016/j.canlet.2011.07.021

Kim MY, Kim EJ, Kim YN, Choi C, Lee BH (2011) Effects of alpha-lipoic acid and L-carnosine supplementation on antioxidant activities and lipid profiles in rats. Nutr Res Pract 5:421-428. doi:10.4162/nrp.2011.5.5.421

Klebanov GI et al (1997) Evidence for a direct interaction of superoxide anion radical with carnosine. Biochem Mol Biol Int 43:99-106

Knorre DA, Severin FF (2012) Longevity and mitochondrial membrane potential. Biochemistry (Mosc) 77:793-794. doi:10.1134/S0006297912070127

Krzepilko A, Swiecilo A, Wawryn J, Zadrag R, Koziol S, Bartosz G, Bilinski T (2004) Ascorbate restores lifespan of superoxide-dismutase deficient yeast. Free Radic Res 38:1019-1024. doi:10.1080/10715760410001717327
Lam YT, Stocker R, Dawes IW (2010) The lipophilic antioxidants alpha-tocopherol and coenzyme Q10 reduce the replicative lifespan of Saccharomyces cerevisiae. Free Radic Biol Med 49:237-244. doi:10.1016/j.freeradbiomed. 2010.04.008

Lin SJ et al (2002) Calorie restriction extends Saccharomyces cerevisiae lifespan by increasing respiration. Nature 418 : 344-348. doi:10.1038/nature00829

McFarland GA, Holliday R (1994) Retardation of the senescence of cultured human diploid fibroblasts by carnosine. Exp Cell Res 212:167-175. doi:10.1006/excr.1994.1132

Medvedik O, Lamming DW, Kim KD, Sinclair DA (2007) MSN2 and MSN4 link calorie restriction and TOR to sirtuin-mediated lifespan extension in Saccharomyces cerevisiae. PLoS Biol 5:e261. doi:10.1371/journal.pbio.0050261

Miceli MV, Jiang JC, Tiwari A, Rodriguez-Quinones JF, Jazwinski SM (2011) Loss of mitochondrial membrane potential triggers the retrograde response extending yeast replicative lifespan. Front Genet 2:102. doi:10.3389/fgene. 2011.00102

Minois N, Frajnt M, Wilson C, Vaupel JW (2005) Advances in measuring lifespan in the yeast Saccharomyces cerevisiae. Proc Natl Acad Sci USA 102:402-406. doi:10.1073/pnas. 0408332102

Mizuno D, Konoha-Mizuno K, Mori M, Sadakane Y, Koyama H, Ohkawara S, Kawahara M (2015) Protective activity of carnosine and anserine against zinc-induced neurotoxicity: a possible treatment for vascular dementia. Metallomics 7:1233-1239. doi:10.1039/c5mt00049a

Molon M, Zadrag-Tecza R, Bilinski T (2015) The longevity in the yeast Saccharomyces cerevisiae: a comparison of two approaches for assessment the lifespan. Biochem Biophys Res Commun 460:651-656. doi:10.1016/j.bbrc.2015.03.085

Mozdzan M, Szemraj J, Rysz J, Nowak D (2005) Antioxidant properties of carnosine re-evaluated with oxidizing systems involving iron and copper ions. Basic Clin Pharmacol Toxicol 96:352-360. doi:10.1111/j.1742-7843.2005.pto_ 03.

Nakaya S, Kobori H, Sekiya A, Kawagishi H, Ushimaru T (2014) Anti-aging and anti-microbial effects of melleolide on various types of yeast. Biosci Biotechnol Biochem 78:455-457. doi:10.1080/09168451.2014.885826

Renner C, Asperger A, Seyffarth A, Meixensberger J, Gebhardt R, Gaunitz F (2010) Carnosine inhibits ATP production in cells from malignant glioma. Neurol Res 32:101-105. doi:10.1179/016164109X12518779082237

Rockenfeller P et al (2015) Phosphatidylethanolamine positively regulates autophagy and longevity. Cell Death Differ 22:499-508. doi:10.1038/cdd.2014.219

Shen Y, Yang J, Li J, Shi X, Ouyang L, Tian Y, Lu J (2014) Carnosine inhibits the proliferation of human gastric cancer SGC-7901 cells through both of the mitochondrial respiration and glycolysis pathways. PLoS One 9:e104632. doi:10.1371/journal.pone.0104632

Snell TW, Fields AM, Johnston RK (2012) Antioxidants can extend lifespan of Brachionus manjavacas (Rotifera), but only in a few combinations. Biogerontology 13:261-275. doi:10.1007/s10522-012-9371-x

Steffen KK et al (2008) Yeast life span extension by depletion of $60 \mathrm{~s}$ ribosomal subunits is mediated by Gen4. Cell 133:292-302. doi:10.1016/j.cell.2008.02.037 
Wanke V, Cameroni E, Uotila A, Piccolis M, Urban J, Loewith R, De Virgilio C (2008) Caffeine extends yeast lifespan by targeting TORC1. Mol Microbiol 69:277-285. doi:10. 1111/j.1365-2958.2008.06292.x

Wierman MB, Smith JS (2014) Yeast sirtuins and the regulation of aging. FEMS Yeast Res 14:73-88. doi:10.1111/15671364.12115

Wu Z, Song L, Liu SQ, Huang D (2014) Tanshinones extend chronological lifespan in budding yeast Saccharomyces cerevisiae. Appl Microbiol Biotechnol 98:8617-8628. doi:10.1007/s00253-014-5890-5

Xie Z, Baba SP, Sweeney BR, Barski OA (2013) Detoxification of aldehydes by histidine-containing dipeptides: from chemistry to clinical implications. Chem Biol Interact 202:288-297. doi:10.1016/j.cbi.2012.12.017

Yuneva AO, Kramarenko GG, Vetreshchak TV, Gallant S, Boldyrev AA (2002) Effect of carnosine on Drosophila melanogaster lifespan. Bull Exp Biol Med 133:559-561

Zadrag R, Bartosz G, Bilinski T (2008) Is the yeast a relevant model for aging of multicellular organisms? An insight from the total lifespan of Saccharomyces cerevisiae. Curr Aging Sci 1:159-165

Zadrag-Tecza R, Molon M, Mamczur J, Bilinski T (2013) Dependence of the yeast Saccharomyces cerevisiae postreproductive lifespan on the reproductive potential. Acta Biochim Pol 60:111-115 